\title{
Identification of multi-exon deletion in the COL7A1 gene underlying dystrophic epidermolysis bullosa by whole-exome sequencing
}

\author{
Mahdieh Taghizadeh', Sima Mansoori Derakhshan², Mahmoud Shekari Khaniani², \\ Yeganeh Eshaghkhani ${ }^{3}$, Zahra Golchehre ${ }^{3}$, Seyedeh Roksana Taheri', \\ Parisa Nourmohammadi', Mohammad Keramatipour ${ }^{1,3}$
}

\begin{abstract}
${ }^{1}$ Watson Genetic Laboratory, North Kargar Street, Tehran, Iran, ${ }^{2}$ Department of Medical Genetics, Tabriz University of Medical Sciences, Tabriz, Iran, ${ }^{3}$ Department of Medical Genetics, School of Medicine, Tehran University of Medical Sciences, Tehran, Iran
\end{abstract}

Corresponding author: Mohammad Keramatipour, MD, E-mail: keramatipour@sina.tums.ac.ir

\begin{abstract}
Dystrophic epidermolysis bullosa (DEB) is a rare form of genodermatosis characterized by skin blisters, milia, scarring over the entire body, and nail dystrophy. In this study, a pedigree with one affected member with skin blisters, and a clinical diagnosis of epidermolysis bullosa who was a result of a non-consanguineous marriage, was investigated by whole-exome sequencing (WES). This survey revealed that the proband is a compound heterozygote for a previously reported heterozygous missense variant (c.6205C > T) and a heterozygous deletion of exons 13-24 in the COL7Al gene. This study indicates that the use of WES along with copy number variation (CNV) analysis gives a higher diagnostic yield for such patients. Moreover, considering the autosomal recessive and dominant forms of the disease, both caused by variants in one gene, proper interpretation and classification of novel variants in heterozygous as well as homozygous states is always a major challenge.
\end{abstract}

Key words: dystrophic epidermolysis bullosa; COL7Al; whole-exome sequencing; deletion; CNV analysis

\section{INTRODUCTION}

Epidermolysis bullosa (EB) is a group of heritable and acquired skin disorders of variable clinical severity. The heritable and severe forms of EB manifest themselves at birth or shortly thereafter as blistering and erosions of the skin and mucous membranes, with considerable morbidity during the early postnatal period, whereas the milder variants are characterized by skin fragility that does not influence the patient's overall lifespan [1]. On the basis of clinical observations and ultrastructural demonstration of the topographic level of blistering within the skin, the hereditary forms of EB have been divided into three categories: epidermolysis bullosa simplex (EBS) involving intra-epidermal blistering with autosomal dominant inheritance; junctional epidermolysis bullosa involving tissue separation within the dermal-epidermal basement membrane with autosomal recessive inheritance; and the dystrophic forms of epidermolysis bullosa involving sublamina densa blister formation within the upper papillary dermis with autosomal dominant or recessive inheritance. There is extensive heterogeneity in the severity of the phenotype and the outcome of the disease in these subgroups of EB. The phenotypic heterogeneity of different forms of $\mathrm{EB}$ reflects the fact that as many as twenty different genes encoding the components of the dermal-epidermal attachment complexes harbor mutations in different subtypes of $\mathrm{EB}$ [2]. Investigation on the genetic aspect of

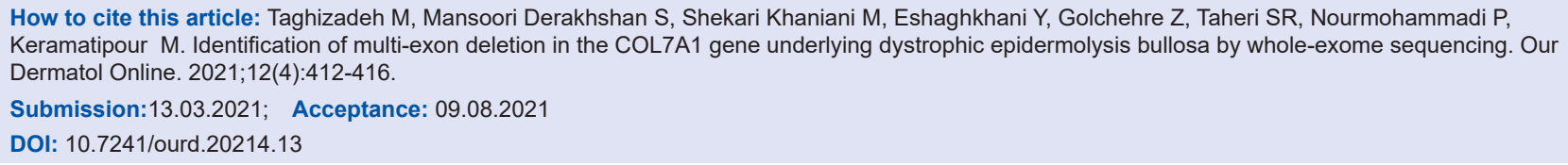


diseases provides the opportunity to explore the relationships between complex phenotypes and genomic variations [3]. Herein, with the help of nextgeneration sequencing (NGS) technology, we report the case of a fifteen-year-old male who had been suffering from chronic skin disease with skin blisters resulting from a compound heterozygote for a previously reported heterozygous missense variant $($ c. $6205 \mathrm{C}>\mathrm{T}$ ) and a novel heterozygous deletion of exons 13-24 in the COL7Al gene.

\section{CASE REPORT}

A pedigree with one affected member was investigated. A fifteen-year-old male with skin blisters, and a clinical diagnosis of epidermolysis bullosa who was a result of a non-consanguineous marriage, was referred to Watson Genetic Laboratory in Tehran, Iran, for genetic counseling and/or analysis. Written informed consent was taken from the patient's parents and peripheral blood samples were collected from the patient and his family (Fig. 1). Genomic DNA was extracted from whole peripheral blood with the GeneAll ${ }^{\circledR}$ Exgene $^{\text {TM }}$ kit (GeneAll Biotechnology Co., LTD, Seoul, Korea) [4]. Human whole-exome enrichment was performed with the Twist Human Core Exome kit and the library was sequenced on the Illumina HiSeq 4000 platform with a raw coverage of $345 \mathrm{X}$ and a mean on-target coverage of 149X, performed by CeGaT GmbH, Germany. Detected variations included single-point mutations and small indels (within 20bp). Furthermore, copy number variation $(\mathrm{CNV})$ detection of the aforementioned genes was performed for the patient with breakpoint analysis. Breakpoint analysis was conducted by longrange PCR followed by Sanger sequencing. Long-range PCR was performed with the TaKaRa PrimeStar GXL kit (TaKaRa Bio Inc., Shiga, Japan). The thermal profile for long-range PCR was as follows: denaturation for $8 \mathrm{~min}$ at $96^{\circ} \mathrm{C}$, then 35 cycles of denaturation $(30 \mathrm{~s}$ at $\left.96^{\circ} \mathrm{C}\right)$, annealing $\left(30 \mathrm{~s}\right.$ at $\left.61^{\circ} \mathrm{C}\right)$, and extension $(4 \mathrm{~min}$ at $72^{\circ} \mathrm{C}$ ), followed by the final extension step of $10 \mathrm{~min}$ at $72^{\circ} \mathrm{C}$. Primers used for genomic amplifications by which the deletion was found were as follows: F, 5'- CCCAGTACCGCATCATTGTG -3' (exon 12); R,

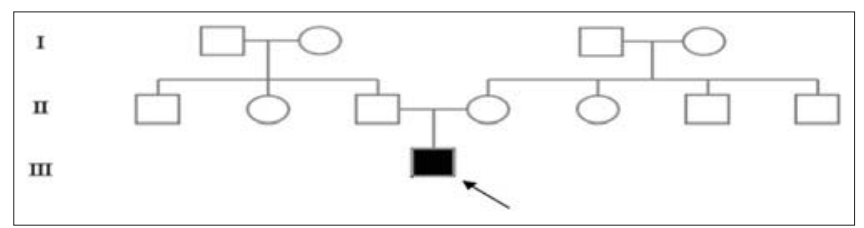

Figure 1: The investigated pedigree, with the proband indicated by filled squares and an arrow.
5'-T GTCACGGATCCTTTGCAAGA - 3' (exon 25). For PCR amplification, $1 \mathrm{ml}(50 \mathrm{ng} / \mathrm{ml})$ of genome DNA was used as a template in $20 \mathrm{ml}$ of reaction mixture, $1 \mathrm{ml}$ of each primer $(5 \mathrm{pM})$, and $8 \mu \mathrm{L} \mathrm{DH} 2 \mathrm{O}$. The PCR products, examined by $2 \%$ agarose gel electrophoresis, were 4,899 bp (for the normal allele) and 836bp (for the mutant allele) in size, respectively. The PCR products were sequenced by an ABI 3500 automated sequencer (Pishgam Biotech Company, Tehran, Iran). Sequences obtained were analyzed with publicly available NCBI Blast, UCSC BLAT, the sequence alignment tool on UCSC (http://genome. ucsc.edu/cgi-bin/hgBlat?). In order to validate the detected missense variant, polymerase chain reaction was followed by Sanger sequencing. The sequences of the designed primers were as follows: forward primer: GAGTGAGGGAAGAGGGGTTG; reverse primer: ACAGGACTAAGGCAGGGATG. PCR reaction was performed in $20 \mu \mathrm{L}$ of the total volume containing $8 \mu \mathrm{L}$ of the Taq DNA Polymerase $2 \times$ Master Mix (Ampliqon A/S, Odense, Denmark), $8 \mu \mathrm{L}$ DH2O, $1 \mu \mathrm{L}$ of each 5 pM primers, and $1 \mu \mathrm{L}$ of $50 \mathrm{ng} / \mu \mathrm{L}$ DNA. The conditions for PCR were as follows: initial denaturation at $96^{\circ} \mathrm{C}$ for 6 min; 32 cycles of denaturation at $96^{\circ} \mathrm{C}$ for $30 \mathrm{~s}$, the annealing step at $60^{\circ} \mathrm{C}$ for $30 \mathrm{~s}$, elongation at $72^{\circ} \mathrm{C}$ for $30 \mathrm{~s}$, and the final extension at $72^{\circ} \mathrm{C}$ for $5 \mathrm{~min}$. The PCR products were sequenced by an ABI 3500 automated sequencer (Pishgam Biotech Company, Tehran, Iran).

Whole-exome sequencing revealed a heterozygous missense variant in the proband. The variant was a C-to-T transition at the first base of codon 2069 in exon 74 of the COL7Al gene that caused the substitution of $\operatorname{Arg}$ (CGT) with Cys (TGT) (Fig. 2). According to our survey, this variant $(\mathrm{c} .6205 \mathrm{C}>\mathrm{T})$ in the COL7Al gene has been reported in the HGMD as well as several other publications as a pathogenic variant [6-11]. The bioinformatic investigation was performed with online tools, including Mutation Taster and CADD, to predict the possible effect of the variant on the function of the protein. The variant was predicted with high confidence to be "disease-causing" by Mutation Taster and with CADD to yield a Phred score of 25.6. The variant is absent in population databases (ExAC, $1000 \mathrm{G})$. In addition, this variant has a frequency of zero in the largest available local database of genomic variations in the Iranian population (Pishgam Biotech Company, Tehran, Iran). Furthermore, a heterozygous deletion of exons 13-24 was detected in the COL7Al gene. $\mathrm{CNV}$ analysis of multiple exon deletions in the 
COL7Al gene for the patient with breakpoint analysis is shown in Fig. 3. Long PCR analysis of genomic DNA indicated 4063 bp deletions of the COL7Al gene in the patient (Fig. 4). This variant had been previously reported for its pathogenicity in homozygous status [5]. The nature of the deletion and multiple lines of in silico computational analysis support the deleterious effect of the variant on the gene or gene product(s). This variant is absent in population databases (ExAC, 1000G) and our local database.

\section{DISCUSSION}

In this study, we attempted to target a group of genes responsible for epidermolysis bullosa to detect causative mutations in an Iranian patient with skin blisters, and a clinical diagnosis of epidermolysis bullosa. As a result, a heterozygous missense variant (c.6205C > T) and heterozygous deletion of exons $13-24$ in the COL7Al gene were detected in the patient. Dominant and recessive mutations in gene COL7Al encoding for collagen VII have been reported to cause DEB. Collagen VII is the major constituent of anchoring fibril presented below the basal lamina within the dermalepidermal junction. Anchoring fibril appears in normal quantity and morphology on biopsy in Dominant DEB (DDEB). In Recessive DEB the amount of collagen VII was usually remarkably reduced on electron microscopy and immunofluorescence staining [12]. Mutations in the COL7Al gene encoding for collagen

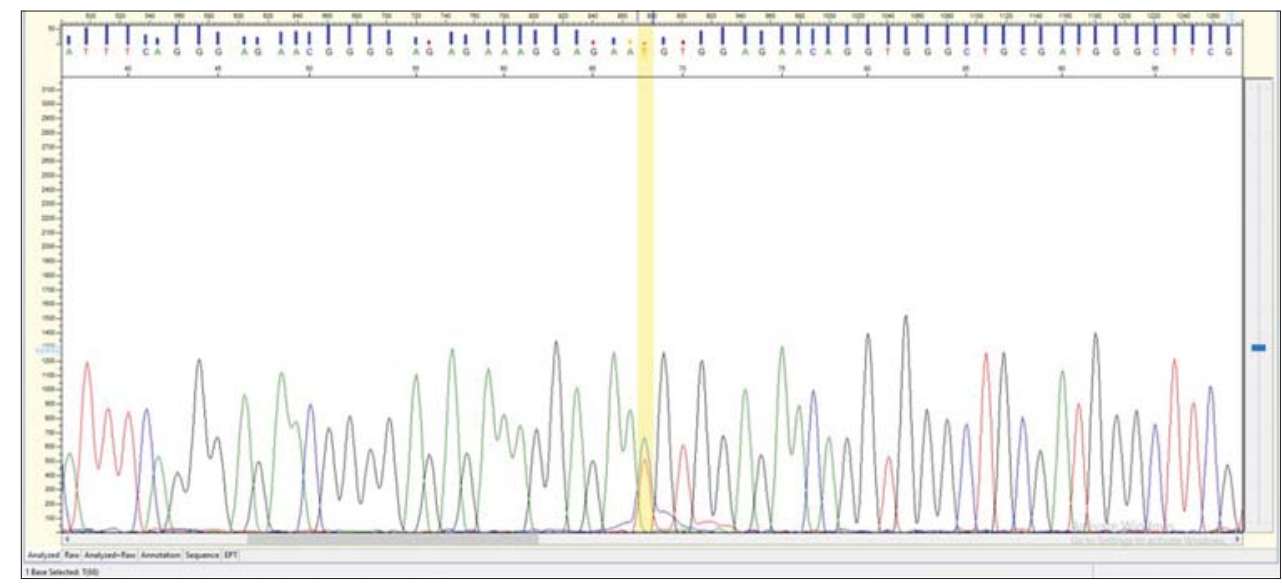

Figure 2: The result of DNA sequencing showing heterozygosity in the patient for variant c.6205C>T in the COL7A1 gene.

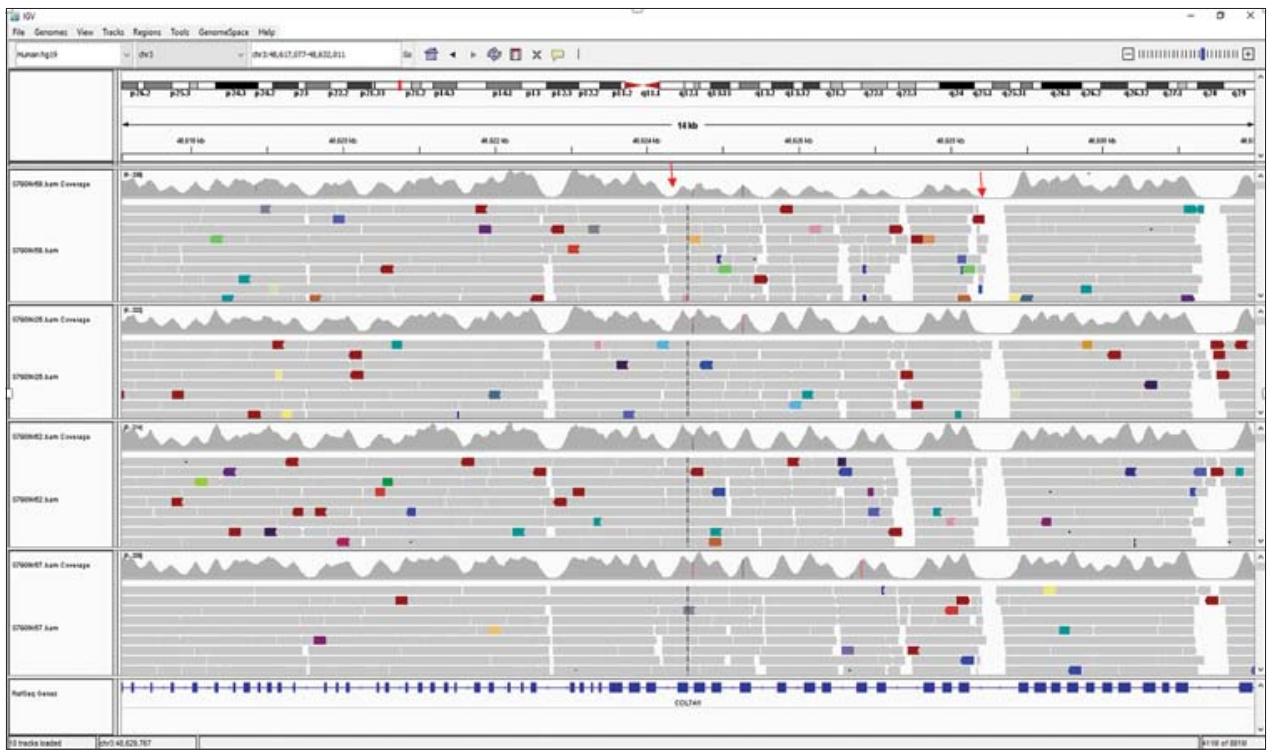

Figure 3: CNV detection of multiple exon deletions in the COL7A1 gene for the patient with breakpoint analysis. Coverage plots (IGV) of three control (ctrl) samples versus the patient (px) sample illustrate the statistical readout with a drop in coverage in the COL7A1 gene on exons 13-24 for the patient sample (double red arrows), compared to controls indicating a deletion event at this position. The 5' decrease in coverage is located at a clear-cut position at the beginning of exon 13 (red arrow). The COL7A1 gene is displayed from the right to left. 


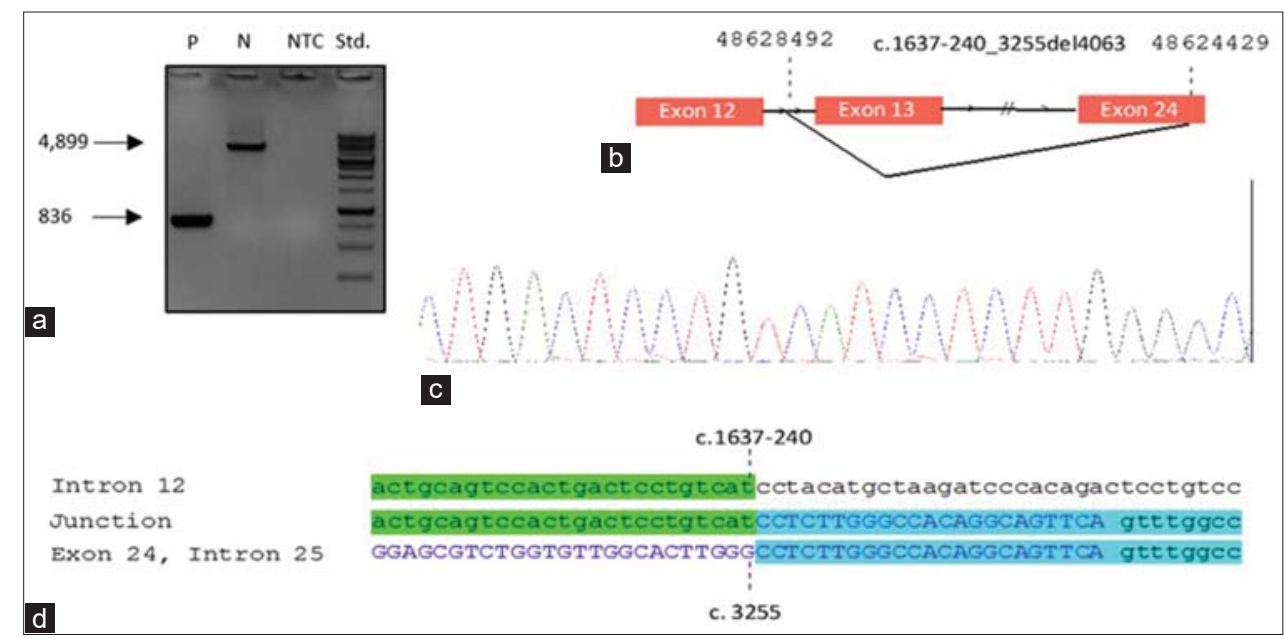

Figure 4: (a) Long PCR analysis of genomic DNA indicating 4063 bp deletions of the COL7A1 gene in the patient. Lane 1 (Std) is a $1 \mathrm{~kb}$ ladder. Lane 2 (NTC) is No Template Control. Lane 3 (Sample N) is amplified from the normal control. Lane 4 (Sample P) points to the abnormal fragments generated by the genomic deletion. (b) Schematic representation of the deletion regions in the COL7A1 gene. (c) The result of DNA sequencing showing heterozygosity in the patient for variant c.1637-240_3255del4063 in the COL7A1 gene. (d) Sequence analysis of junction fragments amplified by PCR revealing deleted sequences within nucleotide (c) at position c.1637-240 in intron 12 and nucleotide (g) at position c. 3255 in exon 24.

VII cause dominant and recessive forms of DEB. Genetic investigations of the COL7Al gene in several patients demonstrated that recessive DEB (RDEB) leads to highly severe phenotypes due to deletions or small insertions and nonsense mutations creating a premature termination codon [13]. On the other hand, the substitution of amino acids causes a dominantly inherited form of DEB with a milder phenotype [14]. Therefore, the EB type was identified by accurate molecular genetic investigation of the COL7Al gene. In the present study, WES sequencing of the patient indicated previously detected heterozygous deletion of exons 13-24, coexisting with heterozygous Single-Nucleotide Variants (SNVs) elsewhere in the COL7Al gene that predicted to be deleterious. We could not confirm compound heterozygosity, as parental DNA was not available, but some studies have suggested an autosomal recessive pattern of inheritance for the c.6205C > T variant [6-8]. In our patient, long-range PCR confirmed the loss of exons 13 to 24 on one allele and a recombination occurring between a short sequence of three nucleotides (CCT) in intron 12 (c.1637-240) and the same sequence at exon 24 (c.3255), resulting in a deletion spanning on 4063 bp (c.1637-240_3255del4063). Descriptions by Vahidnezhad et al. and ours demonstrates that the COL7Al gene, containing numerous repeated sequences, and the affected regions might be prone to recombination. In addition, the geographic location of the family in our study was in the northwest of Iran that was located in the same location indicated in this study [5].

\section{CONCLUSION}

Our study suggests that CNV analysis would be required to reach molecular detection of epidermolysis bullosa. Especially for centers that already employ WES or targeted gene panels, we suggest extending their analysis to include $\mathrm{CNV}$ detection.

\section{ACKNOWLEDGMENTS}

We would like to thank the patient and her family as well as the clinician whose cooperation made this study possible.

\section{Consent}

The examination of the patient was conducted according to the principles of the Declaration of Helsinki.

The authors certify that they have obtained all appropriate patient consent forms, in which the patients gave their consent for images and other clinical information to be included in the journal. The patients understand that their names and initials will not be published and due effort will be made to conceal their identity, but that anonymity cannot be guaranteed.

\section{REFERENCES}

1. Tosti A, Piraccini BM, Scher RK. Isolated nail dystrophy suggestive of dominant dystrophic epidermolysis bullosa. Pediatr Dermatol. 2003;20:456-7.

2. Uitto J, Has C, Vahidnezhad H, Youssefian L, BrucknerTuderman L. Molecular pathology of the basement membrane zone in heritable blistering diseases: The paradigm of epidermolysis 
bullosa. Matrix Biol Plus. 2017;57-58:76-85.

3. Atri Barzanjeh S, Behshid M, Hosseini MB, Ezari M, Taghizadeh M, Dastgiri S. Community genetic services in Iran. Genet Res Int. 2012;2012:129575.

4. Majidzadeh-A K, Zarinfam S, Abdoli N, Yadegari F, Esmaeili R, Farahmand L, et al. A comprehensive reference for BRCA1/2 genes pathogenic variants in Iran: Published, unpublished and novel. Fam Cancer. 2021;1-6.

5. Vahidnezhad H, Youssefian L, Zeinali S, Saeidian AH, Sotoudeh S, Mozafari N, et al. Dystrophic epidermolysis bullosa: COL7A1 mutation landscape in a multi-ethnic cohort of 152 extended families with high degree of customary consanguineous marriages. J Invest Dermatol. 2017;137:660-9.

6. Kern JS, Kohlhase J, Bruckner-Tuderman L, Has C. Expanding the COL7A1 mutation database: Novel and recurrent mutations and unusual genotype-phenotype constellations in 41 patients with dystrophic epidermolysis bullosa. J Invest Dermatol. 2006;126:1006-12.

7. Vendrell X, Bautista-Llácer R, Alberola TM, García-Mengual E, Pardo M, Urries A, et al. Pregnancy after PGD for recessive dystrophic epidermolysis bullosa inversa: Genetics and preimplantation genetics. J Assist Reprod Genet. 2011;28:825-32.

8. van den Akker PC, van Essen AJ, Kraak MM, Meijer R, Nijenhuis M, Meijer G, et al. Long-term follow-up of patients with recessive dystrophic epidermolysis bullosa in the Netherlands: Expansion of the mutation database and unusual phenotype-genotype correlations. J Dermatol Sci. 2009;56:9-18.

9. Woodley DT, Cogan J, Wang X, Hou Y, Haghighian C, Kudo G, et al. De novo anti-type VII collagen antibodies in patients with recessive dystrophic epidermolysis bullosa. J Invest Dermatol. 2014;134:1138-40.

10. Hamidi AK, Moghaddam M, Hatamnejadian N, Ebrahimi A. A novel deletion and two recurrent substitutions on type VII collagen gene in seven Iranian patients with epidermolysis bullosa. Iran J Basic Med Sci. 2016;19:858-62.

11. Dănescu S, Has C, Senila S, Ungureanu L, Cosgarea R. Epidemiology of inherited epidermolysis bullosa in Romania and genotypephenotype correlations in patients with dystrophic epidermolysis bullosa. J Eur Acad Dermatol Venereol. 2015;29:899-903.

12. Yang CS, Lu Y, Farhi A, Nelson-Williams C, Kashgarian M, Glusac EJ, et al. An incompletely penetrant novel mutation in COL7A1 causes epidermolysis bullosa pruriginosa and dominant dystrophic epidermolysis bullosa phenotypes in an extended kindred. Pediatr Dermatol. 2012;29:725-31.

13. Pfendner E, Uitto J, Fine JD. Epidermolysis bullosa carrier frequencies in the US population. J Invest Dermatol. 2001;116:483-4.

14. Nakamura H, Sawamura D, Goto M, Sato-Matsumura KC, LaDuca J, Lee JY, et al. The G2028R glycine substitution mutation in COL7A1 leads to marked inter-familiar clinical heterogeneity in dominant dystrophic epidermolysis bullosa. J Dermatol Sci. 2004;34:195-200.

Copyright by Mahdieh Taghizadeh, et al. This is an open access article distributed under the terms of the Creative Commons Attribution License, which permits unrestricted use, distribution, and reproduction in any medium, provided the original author and source are credited.

Source of Support: Nil, Conflict of Interest: None declared. 